\title{
Zuwanderer als Patienten Kulturelle Vielfalt respektieren
}
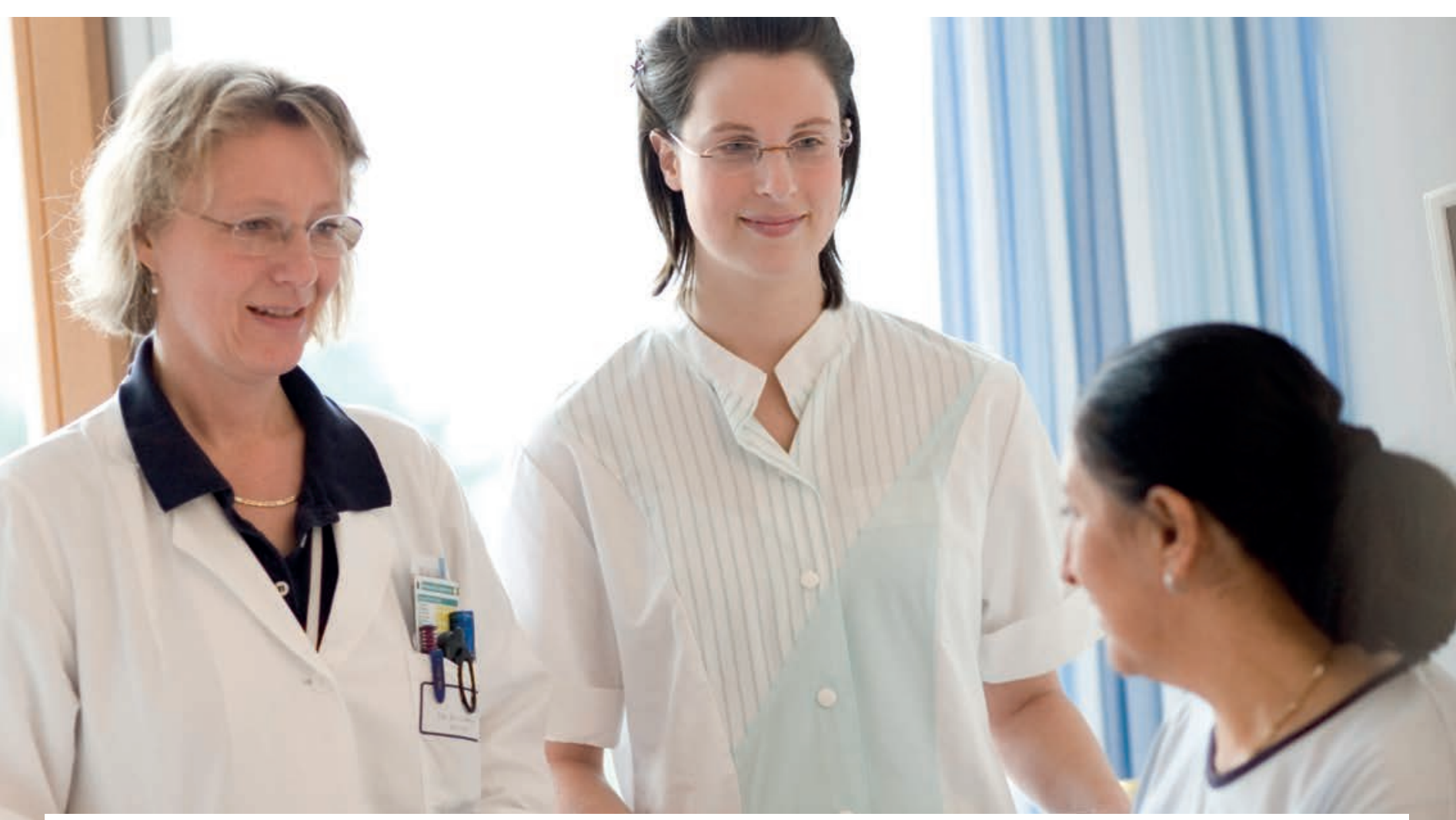

Was tun, wenn Arzt und Patient nicht die gleiche Sprache sprechen und darüber hinaus aus ganz unterschiedlichen Kulturkreisen stammen? Flexibilität und Offenheit für fremde Krankheitskonzepte helfen ein großes Stück weiter, um Patienten mit Migrationshintergrund gerecht zu werden.

Muslimischer Patient - Was fällt Ihnen dazu ein? Diese Frage stellten Mitarbeiter des Lehrbereichs Allgemeinmedizin am Universitätsklinikum Tübingen 90 Hausärzten und baten sie, ihre Assoziationen zu notieren [1]. Die Auswertung der subjektiven Gedanken und Erfahrungen zeigte:

> Bei vielen Befragten dominierte ein negativer Grundtenor. Die Behandlung schätzten sie häufig als schwierig ein. Ursachen dafür waren Verständigungsprobleme, ein andersartiges Krankheitsverständnis und mit Berührungsängsten belastete Untersuchungssituationen.

Aus Sicht der Ärzte beeinträchtigten also v.a. sprachliche und kulturelle Barrieren die Beziehung zu diesen Patienten.
Gute Kommunikation ist essenziell Egal wen Sie behandeln, gute Kommunikation ist eine ärztliche Kernkompetenz [2] - sowohl bei der Anamnese als auch in der Aufklärung über Diagnose und Therapie. Gut zu kommunizieren, bedeutet nicht nur in einem Patientengespräch alle relevanten Informationen zu vermitteln, sondern gleichzeitig sicherzustellen, dass der Patient Sie auch verstanden hat. Genauso wichtig ist jedoch, dass sich der Patient verstanden fühlt. Nur so entsteht Vertrauen in den Arzt, Compliance und damit ein Behandlungserfolg: Probleme in der Kommunikation erhöhen die Wahrscheinlichkeit einer Fehldiagnose und die Anzahl unnötiger Untersuchungen [2]. In einer vertrauensvollen Arzt-Patient-Beziehung treten weniger Komplikationen auf, z.B. weil der Arzt die Fortschritte des Patienten besser erkennt und ein Medikament zum richtigen Zeitpunkt absetzen kann [2]. 


\section{Sprachprobleme: Die Spitze des Eisbergs}

\section{Erhöhter Schwierigkeitsgrad: Fremdsprachige Patienten Ein} erfolgreiches Patientengespräch zu führen, ist schon mit Muttersprachlern nicht einfach: Konflikte können sich allein aus dem Unterschied zwischen Gemeinsprache und Fachsprache des Arztes ergeben. Wer aber weder Deutsch noch eine gängige Fremdsprache wie Englisch spricht, hat es als Patient doppelt schwer. Zum einen kann man sich nicht so leicht verständlich machen. Zum anderen wird man häufig auch nicht richtig verstanden, wie das traurige Beispiel einer 23-jährigen Türkin zeigt. Sie verfügte nur über rudimentäre Deutschkenntnisse und äußerte vor ihrer 2. Entbindung ihrem Arzt gegenüber: „Nix Baby mehr“. Der Arzt informierte sie über Folgen und Bedeutung einer Sterilisation und fragte, ob die Patientin alles verstanden habe. Sie nickte. Im Rahmen des Kaiserschnitts wurde die Sterilisation durchgeführt. Später verklagte die Patienten den Arzt auf Schmerzensgeld: Er habe sie ohne ihr Wissen sterilisiert [3,4].

\section{Sprachbarrieren wirken sich nachweislich negativ auf die gesundheitliche Versorgung aus.}

Praxistipps für das Patientengespräch Was können Sie also tun, wenn Sie einen Patienten behandeln, der nur bruchstückhaft Deutsch spricht?

> Schätzen Sie zunächst seine Sprachkompetenz ab: Offene Fragen eignen sich dazu, einen Eindruck von seinen Deutschkenntnissen zu gewinnen.

> Weniger geeignet sind Fragen wie „Haben Sie mich verstanden?" nachdem Sie eine lange Erklärung gegeben haben oder geschlossene, evtl. sogar suggestive Fragen: „Sie nehmen Marcumar seit 2 Jahren?" [2].

> Bitten Sie den Patienten stattdessen, in eigenen Worten wiederzugeben, was Sie gerade erläutert haben.

> Um sich verständlich zu machen, sollten Sie nicht auf Kindersprache zurückgreifen oder aufhören, Verben zu konjugieren. Einfacher zu verstehen sind kurze, langsam gesprochene Sätze ohne Fachtermini und komplexe grammatikalische Strukturen wie z.B. Relativsätze.

> Achten Sie auch auf eine positive Körpersprache und nutzen Sie eine bildhafte Sprache, Zeichnungen oder ein Zeigewörterbuch. Diffuse Beschwerden können Sie z.B. gemeinsam in einem Anatomieatlas orten.

Wenn Sie langsam sprechen, können Sie außerdem an Mimik, Gestik und Körpersprache Ihres Gegenübers ablesen, wo Verständnis-

\section{So führen Sie ein gedolmetschtes} Patientengespräch $[2,5]$

$>$ Weisen Sie den Dolmetscher auf seine Schweigepflicht hin.

$>$ Bitten Sie ihn, wortwörtlich zu übersetzen.

$>$ Stellen Sie Dolmetscher und Patient einander namentlich vor.

$>$ Erklären Sie dem Patienten, dass der Dolmetscher der Schweigepflicht unterliegt.

> Halten Sie Blickkontakt mit dem Patienten und sprechen ihn direkt an.

> Stellen Sie nur wenige Fragen und geben Sie nur wenige Informationen auf einmal.

> Im Anschluss bitten Sie den Dolmetscher um seine Einschätzung des Gesprächs und bedanken sich für seine Hilfe.

$>$ Ein Good Practice Video finden Sie unter $>$ http://mediathek. hhu.de > Dolmetscher-vermitteltes Arzt-Patienten-Gespräch

schwierigkeiten auftreten [5]. Wertvolle Hinweise können auch die Anworten auf folgende Fragen liefern:

> „Wie nennt man die Beschwerde in Ihrer Muttersprache?“

>,Was hat Ihrer Ansicht nach die Beschwerden verursacht?“ [6]

Patientengespräch zu dritt Bei schwierigen Themen oder wenn Sie nonverbal nicht weiterkommen, lohnt es sich, einen Dolmetscher hinzuzuziehen - manche Krankenhäuser bieten sogar einen professionellen Dolmetscherdienst an. Jemand mit einer professionellen Ausbildung ist in jedem Fall besser geeignet zu übersetzen als Verwandte des Patienten oder Krankenhauspersonal. Unter Umständen bringen Sie Familienangehörige oder Personen aus der gleichen Sprachgemeinschaft nämlich in einen Solidaritätskonflikt: Sie übersetzen dann z. B. schlechte Nachrichten nicht, um den Patienten nicht zu belasten oder um Gepflogenheiten ihrer Kultur nicht zu verletzen [2].

\section{Egal ob Sie auf einen Ad-hoc-Übersetzer oder einen professionellen Dometscher zurückgreifen: Als Arzt bleiben Sie dafür verantwortlich, was Ihr Patient versteht.}

\section{Kultureller Aspekt der Kommunikation}

Missverständnisse trotz gemeinsamer Sprache Auch wenn jemand die Vokabeln einer Fremdsprache perfekt beherrscht, können aufgrund kultureller Differenzen immer noch Verständigungsschwierigkeiten auftreten: In China gilt es z. B. als unhöflich Fragen, Bitten und Ablehnungen direkt auszusprechen. Stattdessen werden sie sehr subtil geäußert, mit der Folge, dass sie ein westlicher Gesprächspartner häufig gar nicht wahrnimmt [7]. Auch nonverbale Kommunikation wie Mimik und Gestik kann sich von Kulturkreis zu Kulturkreis unterscheiden und birgt Potenzial für Konflikte: Während in westlichen Ländern Kopfschütteln „nein“ bedeutet, ist es in Indien mit ,ja“ gleichzusetzen [8]. 


\section{Links zu fremdsprachigen Patienteninfos}

> Verständigungshilfen und Therapiepläne: www.tipdoc.de

$>$ Anamnesebögen:

$>$ www.laek-rlp.de > Behandlung von Asylbewerbern > Anamnesebögen

$>$ www.armut-gesundheit.de > Tipps und Infos

$>$ Impfkalender: www.rki.de > Infektionsschutz > Impfen > Informationsmaterialien in verschiedenen Sprachen

Krankheit ist relativ Gerade die Auffassung von Gesundheit, Kranksein, Unfällen, Krisen, Körper, Bewusstsein, Person, Ätiologie, Verlauf und Ausgang von Erkrankungen ist je nach kultureller Sozialisation unterschiedlich - manchmal sogar innerhalb des Ursprungslands [9]. Das Phänomen Krankheit geht also weit über ein rein biologisch-physiologisch-pathologisches Geschehen hinaus. In muslimischen Kulturen gibt es z. B. die Vorstellung, dass eine Person bei einer anderen durch Anschauen Unheil auslösen kann - der sog. böse Blick [10]. Wie Patienten Symptome beschreiben, unterscheidet sich entsprechend je nach Kulturkreis: Seelisches Leid wird ganz unterschiedlichen Organen zugeschrieben. In Frankreich verbindet man körperliche Beschwerden häufig mit der Leber, in Deutschland dagegen mit dem Herz [10]. Türken betonen v.a. Schmerzen und identifizieren externe Krankheitsursachen wie Schicksal oder Gottes Wille. Sie benutzen außerdem häufig eine bildhafte Sprache, um Krankheiten zu beschreiben, z.B. die Redewendung „In meinem Magen liegt ein Stein“. Allerdings muss diese Aussage nicht auf Magen- oder Verdauungsbeschwerden hinweisen, es kann sich genauso um ein Anzeichen für ein psychisches Leiden handeln. Fragen Sie also in solchen Fällen am besten differenziert nach. Nur so können Sie erkennen, welche kulturspezifische Bedeutung bestimmte Beschwerden Ihres Patienten haben [7].

\section{Um zu einem gemeinsamen Schmerzverständnis zu ge-} langen, eignet sich eine visuelle Analogskala $(\bullet A b b .1)$.

Kulturspezifische Verhaltensweisen Unsere kulturelle Sozialisation prägt nicht nur unsere Auffassung von Krankheit: Auch z.B. zu religiösen Vorschriften, Kleiderordnung, Wohn- und Hygienevorstellungen, familiären Rollenbildern, Trauer- und Bestattungszeremonien gibt es ganz unterschiedliche kulturspezifische Ansichten und Verhaltensweisen, die Konfliktpotenzial bergen können [8].

\section{Müssen Sie Fragen zu möglicherweise kritischen}

Themen stellen, sollten Sie vorher um Erlaubnis bitten:

„Darf ich fragen ...?

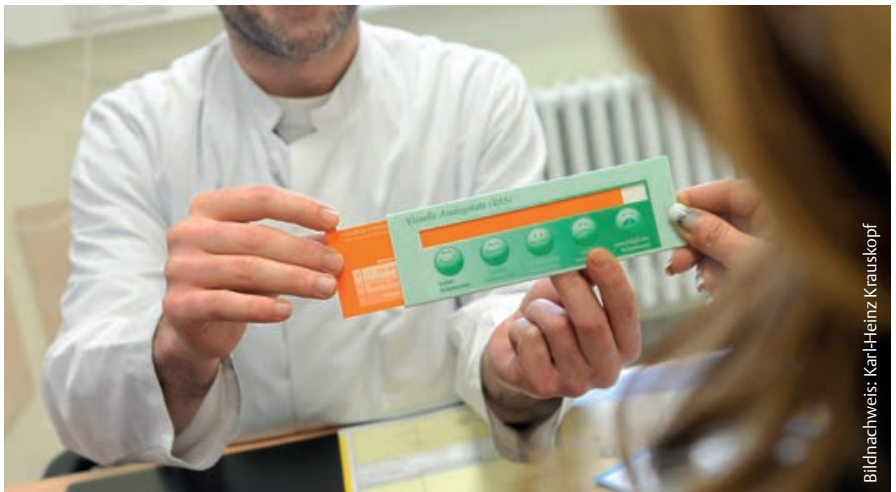

Abb. 1 Eine visuelle Analogskala eignet sich, um das subjektive Schmerzempfinden eines Patienten zu messen.

Schwierig wird es, wenn bestimmte religiöse Pflichten oder Speisevorschriften z.B. im Widerspruch zu therapeutischen Maßnahmen stehen: Zwar lässt der Islam bei Kranken Ausnahmen zu, doch ein gläubiger Muslim könnte die Einnahme von Medikamenten während des Fastenmonats Ramadan verweigern oder Medizinprodukte ablehnen, die nach islamischen Vorschriften verbotene Inhaltsstoffe enthalten (Alkohol oder Schweineprodukte wie Gelatine bei Kapseln). Gegebenenfalls können Sie in solchen Fällen den örtlichen Imam um Vermittlung bitten [6].

Patienten aus islamischen Ländern sind 2 weitere Aspekte besonders wichtig: das Schamgefühl, das den Schutz des Körpers vor Blicken und dem Körperkontakt mit Fremden fordert, und die körperliche Unversehrtheit. Während in westlichen Ländern der Händedruck Offenheit symbolisiert, kann er bei muslimischen Patienten als Verletzung der körperlichen Integrität gelten [7].

\section{Meiden Sie sozialen Körperkontakt, mit dem Sie Ihren Patienten in Verlegenheit bringen könnten.}

Stammt ihr Patient aus einer Kultur, die eine strikte Geschlechtertrennung verlangt, sollte ihn nach Möglichkeit ein Mitarbeiter gleichen Geschlechts betreuen oder zumindest bei der Untersuchung anwesend sein [6].

\section{Interkulturelle Kompetenz als Lösung?}

„Den“ Migranten gibt es nicht Krankenhäuser, in denen kulturelle Unterschiede in der medizinischen Versorgung berücksichtigt werden, z.B. mit mehrsprachigen Informationsmaterialien ( Infokasten oben), entsprechendem Speiseangebot oder kulturspezifischem Belegungsmanagement, reduzieren Fehldiagnosen und Liegezeiten. Außerdem haben sie zufriedenere Patienten und Mitarbeiter [10]. Andererseits ist es natürlich nahezu unmöglich, auf sämtliche kulturspezifische Merkmale einzugehen. Und darüber hinaus mögen sich zwar Migranten von Menschen ohne Migrationshintergrund unterscheiden, aber: „Den“ Migranten gibt es ebensowenig wie z.B. „den“ Muslim. Individuelle Biogra- 


\section{Interkulturelle Kompetenz [12, nach 13]}

„Zusammengefasst beinhaltet der Begriff, interkulturelle Kompetenz' in der Gesundheitsversorgung vor allem Sensibilität für die im Einzelfall relevanten soziokulturellen Aspekte von Krankheit, medizinisch-therapeutischen Maßnahmen und insbesondere der sozialen Interaktion zwischen medizinischem Fachpersonal und Patienten bei augenscheinlich bestehenden kulturellen und / oder ethischen Unterschieden. Kenntnisse über ,fremde Kulturen' sind dabei nur insoweit sinnvoll, wie sie zum Nachdenken und im konkreten Einzelfall zu respektvollem Nachfragen Anlass geben und mit der notwendigen Skepsis gegenüber Verallgemeinerungen und Stereotypien einhergehen. Nicht umfassendes Faktenwissen, welches im Sinne einer Festplatten-Metapher vom Nutzer im Bedarfsfall ,abgerufen' wird, sondern die so genannten ,soft skills' wie Reflexions- und Kommunikationskompetenz sowie Empathie und die Fähigkeit zum Perspektivenwechsel sind die zentralen Aspekte von ,interkultureller Kompetenz' in der Medizin.“

fien, Erfahrungen wie Flucht, Trennung, Traumatisierung und soziokulturelle Isolation, Lebensverhältnisse, Bildung, Ziele, Handlungsmuster und vieles mehr beeinflussen das Krankheitsbild und die medizinische Behandlung [11]. Nutzen einem Arzt also überhaupt Kenntnisse zu unterschiedlichen Krankheitsvorstellungen und Länderinformationen?

Sind mehrsprachige Patienteninformationen sinnvoll? Die Autoren der „Handreichung Interkulturelle Kompetenz in Kliniken“ hinterfragen, ob wirklich kulturspezifische Krankheitskonzepte zu Missverständnissen führen und suchen die Ursache eher bei folgenden Faktoren:

$>$ Zeitmangel des Personals

$>$ Kommunikationsdefizite

$>$ das Denken in Stereotypen

$>$ fehlende Kenntnisse des deutschen Gesundheitssystems auf Seiten der Migranten [12]

In den Augen der Autoren sind mehrsprachige Patienteninformationen und religionsneutrale Andachtsräume zwar hilfreich, aber als alleinstehende Maßnahmen lediglich Trostpflaster, wenn es in einem Krankenhaus keine umfassende Strategie gibt, die Gesundheitsversorgung von Patienten mit Migrationshintergrund zu optimieren. Insbesondere sollte das Personal in interkultureller Kompetenz geschult sein ( Infokasten oben). Fortbildungsangebote bietet z.B. das Projekt „fit for diversity skills“ ( http:// fit-for-diversity-skills.de)

Kultursensibel behandeln - was heißt das konkret? Interkulturelle Kompetenz wird häufig in einem Atemzug genannt mit Kultursensibilität. Beide Begriffe zielen auf einen konfliktfreien Umgang mit unbekannten Verhaltensweisen ab:

> Versuchen Sie, kulturelle Unterschiede zu erkennen, ohne darüber zu urteilen.

\section{Fazit}

Um Patienten mit Migrationshintergrund für alle Beteiligten zufriedenstellend zu behandeln, hilft es, über die fremde Kultur Bescheid zu wissen. Viel wichtiger ist es jedoch, auf die individuellen Wünsche und Erwartungen des Patienten einzugehen, ggf. mit Verständigungshilfen oder einem Dolmetscher, um Sprachbarrieren zu überwinden.

> Häufig hilft ein Perspektivwechsel: Könnte als aggressiv wahrgenommenes Verhalten auch Ausdruck von Hilflosigkeit, Schuldgefühlen, Trauer oder Wut sein?

> Gehen Sie empathisch vor und lassen Sie kulturelle Annahmen ggf. beiseite.

> Suchen Sie nach Gemeinsamkeiten als Verständigungsbrücke. Angst, Verzweiflung und Schmerz sind universell.

$>$ Fragen Sie sich: „Wie würde ich mich in dieser Situation fühlen?" [8]

Die Autoren der eingangs geschilderten Studie bemängeln die Assoziationen der befragten Hausärzte zu „muslimischer Patient“ als zu wenig differenziert und oft stereotypisierend [1]. Die Ursache sehen sie darin, dass die Behandelnden zu wenig über Religion und Kultur der als schwierig eingeschätzten Patienten wissen. Häufig würden diese als homogene Gruppe wahrgenommen, die sich von anderen Patientengruppen als problematisch abhebe. Für ein besseres Verständnis ist es wichtig, ein Gefühl für die Lebenshintergründe des individuellen Patienten zu bekommen. Das erreicht man sicherlich eher, indem man einem Patienten zuhört, als wenn man sich detaillierte Kenntnisse seines kulturellen Hintergrunds aneignet - vorausgesetzt der Praxis- und Klinikalltag bietet dafür Zeit.

Julia Hecht

\section{Literatur}

1 Kronenthaler A, Hiltner H, Eissler M. Assoziationen zu muslimischen Patienten in der Hausarztpraxis - Eine Befragung deutscher Allgemeinmediziner. Gesundheitswesen 2014; 76: 434-439

2 ÄK Nordrhein. Kommunikation im medizinischen Alltag - Ein Leitfaden für die Praxis. Düsseldorf: Ärztekammer Nordrhein; 2015

e Literatur online Das vollständige Literaturverzeichnis zu diesem Beitrag finden Sie im Internet: Unter, www.thieme-connect. de/products “ können Abonnenten und Nichtabonnenten die Seite der Lege artis aufrufen und beim jeweiligen Artikel auf „Ergänzendes Material“ klicken - hier ist die Literatur frei zugänglich.

Lesen Sie dazu auch Unser Interview mit Dr. Simon Reichenauer auf der folgenden Seite sowie „Muslimische Patienten: Bedürfnisse erkennen und respektieren“ von Rojahn J. in der Lege artis (3/2011) Seite 162-166. Für Abonnenten kostenlos: Rufen Sie unter www.thieme-connect.de/ products die Seite der Lege artis auf und wählen die jeweilige Ausgabe. 


\section{„Die Flüchtlinge sind einfach dankbar!“}

\section{Interview mit Dr. Simon Reichenauer, Anästhesist}

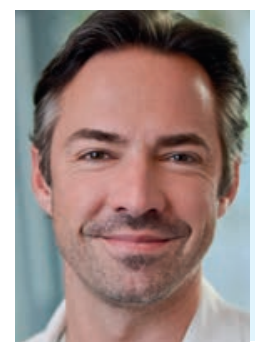

\section{Dr. med. Simon Reichenauer}

ist Facharzt für Anästhesie, Notfall-, Palliativ- und Intensivmedizin in der interdisziplinären Notaufnahme (INA) am Katharinenhospital, Stuttgart. Gemeinsam mit Kollegen organisiert er die Hausarztbetreuung von Flüchtlingen an 2 Standorten. E-Mail: s.reichenauer@klinikum-stuttgart.de

Im Oktober 2015 haben Sie und Ihre Kollegen begonnen, die hausärztliche Versorgung von Flüchtlingen in Stuttgart zu organisieren. Wie kam es dazu?

Unser Projekt ist aus der Not heraus geboren worden. Auf einen Schlag kamen damals Busladungen von Flüchtlingen an, die zunächst notdürftig am Hauptbahnhof untergebracht wurden. Es gab vor Ort nur einen Sanitätsdienst, anfangs vom DRK und den Maltesern. Deren Mitarbeiter durften aber keine Medikamente verabreichen. Alles, was sich nicht mit einem Pflaster und gut zureden behandeln ließ, haben sie in die Krankenhäuser geschickt. Unser Klinikum liegt in der Nähe des Bahnhofs, sodass wir morgens häufig 20 Flüchtlinge in der Notaufnahme hatten. Das waren aber keine Notfälle, sondern sie kamen v.a. mit posttraumatischen Belastungsstörungen, Hautkrankheiten, respiratorischen Infekten oder chronischen Schmerzen.

Hätte man das nicht mit einer ambulanten hausärztlichen Versorgung regeln können?

Ja, aber als wir nachgeforscht haben, stellte sich heraus, dass sich noch niemand um die Flüchtlinge kümmerte. Man könnte sagen, es war ein „medizinfreier Raum“: Keiner hat sich so richtig berufen gefühlt, und es war auch rechtlich niemand verantwortlich, weil das Land für die nicht registrierten Flüchtlinge in den Notunterkünften zuständig ist. Und das Land selbst hat ja keine Medizineinrichtung bis auf die Gesundheitsämter, die natürlich nicht vor Ort eine Sprechstunde anbieten können. Erst als Asylbewerber fallen die Flüchtlinge in die Verantwortung der Kommunen oder Gemeinden und damit der Hausärzte. Wir haben uns dann mit dem Regierungspräsidium, den Maltesern, dem DRK und den Johannitern kurzgeschlossen. So kam das langsam ins Rollen.

\section{Wie sah die Hilfe konkret aus?}

Anfangs haben wir von den Maltesern, die in Stuttgart den hausärztlichen Notdienst organisieren, ein Fahrzeug geliehen bekommen. Damit sind wir dann gemeinsam morgens in die Flüchtlingsunterkünfte gefahren und haben eine Sprechstunde abgehalten, zunächst täglich, später nach Bedarf 2-3 × pro Woche. Zunächst hatten wir nur ein paar provisorische Bierbänke als Sitzmöglichkeit und Trennwände als Sichtschutz.

\section{Und wie versorgen Sie Ihre Patienten heute?}

Bei den Flüchtlingsunterkünften im Neckarpark haben wir jetzt einen eigenen kleinen Container mit 2 Behandlungszimmern. Wir können Blutdruck messen und EKG anfertigen, Ohren spiegeln, haben Mundspatel und Stethoskope, außerdem die ganzen Verbrauchsmaterialien, die man im Alltag so benötigt. Ein Hersteller hat uns sogar ein Ultraschallgerät zur Verfügung gestellt. Mittlerweile wird eine richtige Praxis daraus! Täglich von 9-13 Uhr ist Sprechstunde. Wenn wieder auf einen Schlag 500 Flüchtlinge ankommen, wissen wir, dass eine Woche lang viel los sein wird. Dann schicken wir 2 Ärzte hin, ansonsten reicht einer. Im Moment sind außerdem rund um die Uhr 2 Mitarbeiter von den Hilfsorganisationen vor Ort - entweder Rettungssanitäter oder Rettungsassistenten.

\section{Wie läuft die Versorgung mit Medikamenten?}

In den ersten paar Tagen stammten die Medikamente noch aus dem Vorrat der Hilfsorganisationen, bis wir das mit dem Regierungspräsidium geklärt hatten. Dann konnten wir mit einer Apotheke zusammenarbeiten, die von uns Privatrezepte bekommen und diese mit dem Regierungspräsidium abgerechnet hat. Das war logistisch natürlich etwas aufwendig, aber da die Patienten ja nicht registriert sind und wir nicht einmal einen Namen hatten, geschweige denn eine Kranken- oder Sozialversicherungsnummer, mussten wir etwas unorthodox vorgehen. Mittlerweile haben wir einen eigenen Medikamentenvorrat: Schmerzmittel, Antibiotika auch Säfte und Zäpfchen für die Kinder. Einzelne Verordnungen machen wir immer noch auf Rezept, die die Apotheke dann ans Regierungspräsidium schickt.

\section{Welche Erkrankungen behandeln Sie am häufigsten?}

Eigentlich das gesamte Hausarztspektrum: respiratorische Infekte, Bronchitiden, Laryngitiden, Pneumonien, aber auch Bluthochdruck, Diabetes oder Schwangerschaftstestungen. Viele Flüchtlinge kommen auch wegen Schmerzen durch Verletzungen oder chronische Leiden. Natürlich sehen wir auch Krankheiten, die 


\section{Hausarztbetreuung für Flüchtlinge}

Dr. Simon Reichenauer, Dr. Christian Menzel und Dr. Stefan Rauscher, Oberärzte der Interdisziplinären Notaufnahme am Katharinenhospital Stuttgart, betreuen seit Oktober 2015 in der Nähe der Cannstatter Wasen eine Zelt- und Containerstadt mit 1000 Flüchtlingen - voraussichtlich bis Juni 2016. Anfang des Jahres kam eine weitere Notunterkunft für 1000 Flüchtlinge dazu, die auf 3-4 Jahre ausgelegt ist. Reichenauer und seine Kollegen betreuen die Flüchtlinge ca. 2-6 Wochen lang, bis diese in eine Landeserstaufnahmestelle (LEA) verlegt werden. In den Unterkünften wohnen überwiegend Männer, aber auch viele Familien mit Kindern aller Altersstufen. Vor allem Letztere und Schwangere machen den Großteil der Patienten aus. gefunden, die die Flüchtlinge behandeln. Mittlerweile haben wir einen kleinen Stamm von ungefähr 10 Praxen. Da rufen wir einfach an und machen einen Termin aus, dann werden die Patienten am nächsten Tag mit dem Taxi dorthin gefahren, der Dolmetscher kommt gleich mit.

Kommen Krankheiten vor, die Sie sonst nicht in Deutschland behandeln?

Gerade Skabies haben bei uns wohl die wenigsten Ärzte schon gesehen, ich kenne sie auch nur aus dem Ausland. Dann kommen natürlich noch Kriegsverletzungen und Entstellungen dazu. Bisher hatten wir 2 Tuberkulosen, aber die gibt's bei uns ja auch.

\section{Wie viele Patienten behandeln Sie?}

Ungefähr 6-8\% der Flüchtlinge kommen zu uns in die Sprechstunde, das macht pro Tag etwa 20-30 Kontakte. Wenn ein neuer Schub ankommt, behandeln wir 50-70 Patienten am Tag - wer drankommen möchte, kommt auch dran, dann dauert die Sprechstunde eben länger.

\section{Sehen Sie Patienten öfter als einmal?}

Viele Flüchtlinge betreuen wir kontinuierlich. Wir haben sogar ein eigenes Dokumentationssystem, das eigentlich für einen Massenanfall von Verletzten konzipiert ist, aber vom Hersteller an unsere Situation angepasst wurde. Jeder Patient erhält ein Barcode-Armband, das sich einscannen lässt. So hat man sofort Zugriff auf die ganze Krankengeschichte und darauf, was die Kollegen verordnet haben. Wir möchten jetzt auch mit einem Impfprogramm anfangen, das erleichtert unsere Arbeit natürlich deutlich.

\section{Machen die Ärzte aus freien Stücken mit?}

Es läuft alles auf freiwilliger Basis, die einzigen, die von uns eingeteilt wurden, sind die Ärzte der Notaufnahme. Aber keiner hat bisher etwas dagegen gehabt, und für sie ist es auch nichts anderes als in der Notaufnahme. Im Gegenteil, die meisten freuen sich über die Abwechslung. Anfangs sind wir davon ausgegangen, dass wir es ehrenamtlich machen, aber das Regierungspräsidium hat uns schnell zugesichert, dass der Einsatz als Überstunden bezahlt wird. Seit Dezember sind die Stunden meiner Kollegen der INA sogar in den Dienstplan integriert - das ist natürlich eine Ausnahme.

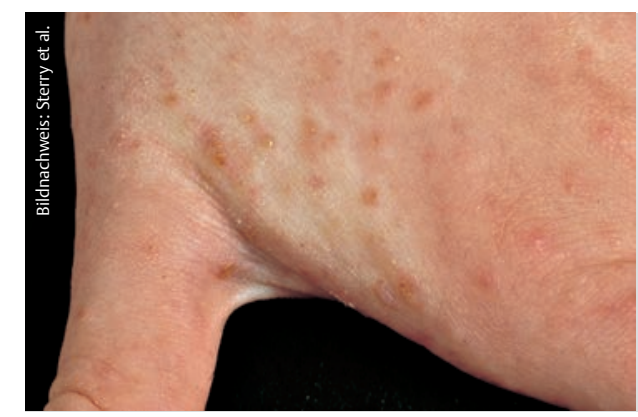

Abb. Skabies mit Milbengängen und entzündlichen Papeln im Interdigitalbereich. 


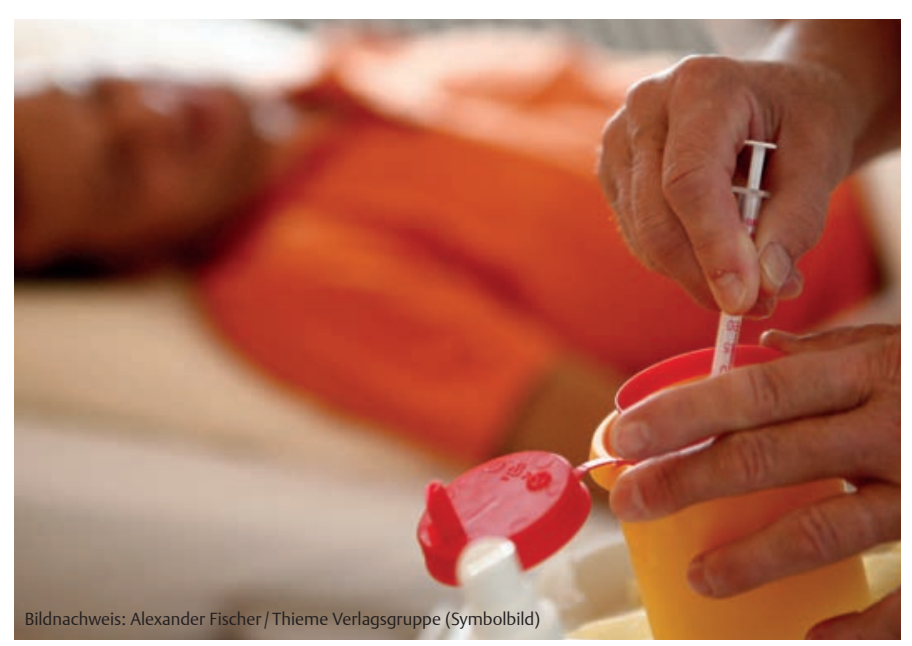

besser auskennt. Wenn es akut ist, können wir die Patienten auch in die Notambulanzen verweisen. Am Klinikum Bad Cannstatt gibt es außerdem eine Hautklinik, die auch schon Patienten übernommen hat.

Die Flüchtlinge stammen v.a. aus Syrien, Afghanistan und Pakis$\tan$ - wie funktioniert die Kommunikation?

Anfangs war es etwas schwierig: Wir haben oft über andere Flüchtlinge kommuniziert, viele von ihnen können sehr gut Englisch. Trotzdem weiß man natürlich nicht so genau, was bei den Patienten ankommt, nicht alle Flüchtlinge sprechen den gleichen Dialekt. In der ersten Zeit war das wirklich ein Problem, das die Sprechstunde deutlich in die Länge gezogen hat. Manchmal kam ein Redeschwall vom Patienten, den der Dolmetscher nur mit einem einzigen Wort, z.B. Fieber wiedergegeben hat. Man wusste also genau, da fehlt jetzt irgend etwas. Inzwischen läuft die Kommunikation aber richtig gut. Das Unternehmen Campanet stellt Dolmetscher, und auch beim Sicherheitsdienst sprechen alle neben Deutsch eine der Sprachen der Flüchtlinge. Häufig sind auch unter den Flüchtlingen selbst Ärzte. Gerade helfen uns ein Kinderarzt und eine Gynäkologin in der Sprechstunde. Sie dürfen in Deutschland zwar nicht selbst praktizieren, aber sie können übersetzen. Das macht den Zugang zu den Patienten deutlich einfacher. Wir haben auch Zeigewörterbücher, aber ich habe sie noch nicht benutzt, weil die Dolmetscher einfach sehr gut sind.

Gibt es kulturelle Barrieren, die die Behandlung erschweren? Manchmal, aber ich habe eigentlich mit mehr Problemen gerechnet. Häufig kommen Frauen in Begleitung ihres Mannes, der dann auch bei der Untersuchung dabei ist. Ein oder zwei der Frauen wollten sich auch nicht von einem Mann untersuchen lassen. Aber da konnten wir sie auf den nächsten Tag verweisen, an dem eine Kollegin da war. Es hat sich bisher auch noch kein Mann dagegen gewehrt, dass ihn eine Ärztin behandelt. Ich glaube, dass die Flüchtlinge in ihrer Not einfach froh sind, endlich einen sicheren Hafen gefunden zu haben. Sie möchten, dass wir ihnen helfen, und respektieren dann auch unsere Gepflogenheiten.
Welcher Aspekt der Versorgung fordert Sie am meisten?

Die unglaubliche Vielfalt der Patienten ist etwas, das eigentlich keine Disziplin so richtig gewohnt ist, nicht einmal die Allgemeinmediziner: Männer, Frauen, Kinder, Schwangere. Dazu kommt das Spektrum an Krankheiten, die man im Alltag vielleicht nicht so häufig sieht, gerade Haut- und Geschlechtskrankheiten. In unserem spezialisierten Gesundheitssystem bekommen wir als Fachärzte viele Patienten gar nicht mehr zu Gesicht, weil sie z.B. gleich zum Gynäkologen gehen.

\section{Was machen Sie heute anders als zu Beginn?}

Es gibt viele Kleinigkeiten, die wir erst einmal lernen mussten. Zum Beispiel: Wer spricht zuerst mit dem Patienten? Anfangs waren es z.T. die Hilfsorganisationen, jetzt werden alle Patienten direkt vom Arzt angeschaut. Den Bedarf an Arzneimitteln mussten wir natürlich erst anpassen. Welche Cremes und Waschlotionen zur Läuse- und Skabiesbehandlung am besten geeignet sind, mussten wir herausfinden. Nach und nach haben wir auch mitbekommen, dass es besser ist, den Patienten häufger ein paar Tabletten mitzugeben als einmal eine Großpackung. Sonst werden Schmerzmittel undifferenziert an andere weitergegeben. In den Herkunftsländern der Flüchtlinge ist der Zugang zu Medikamenten eben nicht selbstverständlich.

\section{Wie lange wird das Projekt fortgesetzt?}

Am Anfang haben wir gedacht, es geht um einige Wochen, dann wurden Monate daraus. Mittlerweile haben wir uns auf Jahre eingestellt. Das ist natürlich eine ganz andere Größenordnung. Für 2016 hat uns das Regierungspräsidium aber eine halbe Stelle zugesichert, die wir für die Organisation und für die Sprechstunde unter dem Notaufnahmeteam aufteilen. Wir möchten das Projekt auf jeden Fall fortsetzen: Mittlerweile kennen wir alle wichtigen Ansprechpartner, die zeitliche Belastung ist nicht mehr ganz so groß, es läuft einfach. Vor allem sind die Patienten unglaublich dankbar und freundlich, es macht unheimlich Spaß, sie zu behandeln!

Die Fragen stellte Julia Hecht. 